\title{
Complex atomic fine structure in the phase domain: exciting opportunities and challenges
}

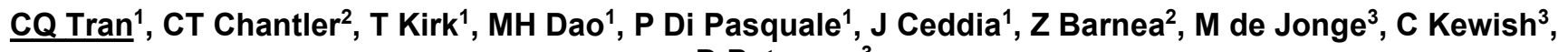 D Paterson ${ }^{3}$}

${ }^{1}$ Department of Chemistry and Physics, School of Molecular Sciences, La Trobe University, Victoria, Australia; ${ }^{2}$ School of Physics, The University of Melbourne, Victoria, Australia, ${ }^{3}$ XFM beamline, Australian Synchrotron, ANSTO, Victoria, Australia;

cq.tran@latrobe.edu.au

X-ray Absorption Spectroscopy has been one of the most powerful tools for probing atomic and molecular structures of materials. However, the measured fine structures in the absorption domain do not have adequate dimensionalities to extract three-dimensional structural information of the material of interest. A technique that allows accurate measurements of atomic fine structure in both the absorption and phase domains will open exciting opportunities in a wide range of fundamental and applied research. In this presentation, we will describe a new technique for determining simultaneously the real and imaginary components of the complex atomic form factor. The technique used Fourier Transform Holography with an extended reference and applicable to both crystalline and amorphous samples. Details of an application of the technique in spectroscopy mode to obtain the X-ray Complex Fine Structure across the copper K-edge will be discussed.

Keywords: Atomic Form Factor; X-ray Absorption Fine Structure; X-ray Complex Fine Structure; Fourier Transform Holography 